\title{
Investigadores y publicaciones científicas en la UNAH
}

Researchers and scientific publications at UNAH

DOI 10.5377pc.v0i16.8091

Nitzia J. Vasquez Alvarado ${ }^{1}$

\section{RESUMEN}

Desde hace algún tiempo, la Universidad Nacional Autónoma de Honduras cuenta con políticas para la mejora de la calidad de los procesos en la investigación científica, siendo esta una de las tres funciones más importantes de la universidad. En este contexto, la Dirección de Investigación Científica, como instancia de gestión general de la investigación de la UNAH, utiliza la Política de Investigación Científica, Desarrollo e Innovación, como herramienta guía para la creación e implementación de mecanismos que contribuyan a la consecución de objetivos en materia de investigación científica. La política cuenta con varios ejes orientadores, entre ellos, la publicación de la producción científica. Cada uno de los cinco ejes de la política comparten actividades que permiten la operación de los procesos que están orientados a estimular y fortalecer la actividad de investigación en la Universidad, así como su difusión en distintos medios académicos a nivel nacional e internacional. El presente artículo pretende caracterizar algunas de las actividades en torno a la publicación científica de los últimos años en la UNAH.

Palabras clave: Investigación científica, publicación científica, universidad pública.

1 Jefe de departamento de Documentación e Información, Dirección de Investigación Científica Universitaria, DICU-UNAH: investigacionunah.informa.nv@gmail.com, nvasquez@unah.edu.hn 


\section{ABSTRACT}

For some time, the National Autonomous University of Honduras has created policies for improving the quality of scientific research processes, this being one of the three most important functions of the university. In this context, the Directorate of Scientific Research, as an instance of general management of UNAH research, uses the Policy of Scientific Research, Development and Innovation as a guiding tool for the creation and implementation of mechanisms that contribute to the achievement of objectives, in the field of scientific research. The policy has several guiding axes, among them, the publication of scientific production. Each of the five axes of the policy share activities that allow the operation of the processes that are oriented to stimulate and strengthen the research activity at the University, as well as its diffusion in different academic media at a national and international level. The present article pretends to characterize some of the activities around the scientific publication of the last years in the UNAH.

Keywords: Scientific research, scientific publication, public university. 


\section{INTRODUCCIÓN}

La Política de Investigación, Desarrollo e Innovación de la UNAH es el instrumento que guía los esfuerzos para el impulso, fortalecimiento y consolidación del desarrollo de la ciencia y la tecnología como pilar fundamental de la institución; ese documento establece dentro de sus ejes orientadores, la publicación, eje que apunta a la difusión exitosa y competitiva de los resultados de la producción investigativa a través de revistas y libros científicos. A continuación mencionaremos algunas estrategias que la Dirección de Investigación Científica Universitaria (DICU) ha puesto en marcha para la publicación de la investigación científica en la institución, así como de algunos de los retos y desafíos que en la actualidad enfrenta en estos ámbitos.

\section{La investigación científica como medio de difusión y divulgación}

Las universidades públicas cuentan con una limitada disposición de recursos para desarrollar la investigación científica. Es por ello que la Universidad Nacional Autónoma de Honduras (UNAH) ha definido claramente trece temas de investigación, tomando como base las líneas de investigación del Consejo Superior Universitario Centroamericano (CSUCA) y el Plan de Nación del Estado de Honduras. La UNAH a través de la Dirección de Investigación Científica, ha destinado recursos financieros para la promoción de la investigación científica, tal es el caso de las becas de investigación científica y la asignación como carga académica. Becas de investigación, en base a fondos concursables cuya convocatoria será pública y bianual. Incluye el otorgamiento de becas sustantivas, básicas, tesis de posgrado para profesores y becas para estudiantes de grado y posgrado, y otras modalidades que se vayan incorporando. (DICYP, 2015). A partir del año 2012, se conoce la "asignación de investigación como carga académica", la cual brinda la posibilidad de que los profesores/investigadores puedan dedicar parte de su jornada laboral al desarrollo de actividades de investigación, pero se encuentra supeditada a las necesidades de la unidad académica a la que se adscribe y a la aprobación de un proyecto específico de investigación sujeto a seguimiento por parte de la DICU.

\section{Publicación científica}

La producción científica desarrollada a través de becas de investigación y de la asignación de la investigación como carga académica, se difunde generalmente a través de artículos científicos. Las revistas científicas permiten llevar un proceso de 
evaluación de la calidad de los avances en las diferentes disciplinas en las que se investiga.

La DICU edita semestralmente 2 revistas científicas: Ciencia y Tecnología y Portal de la Ciencia. Ciencia y Tecnología, publicación que incluye artículos de diversas disciplinas académicas producidos por investigadores profesionales, ha tenido una afluencia mayor de publicación de artículos del área de las Ciencias Biológicas y de la Salud. (Gráfico 1). Portal de la Ciencia, la cual está destinada a la publicación de artículos científicos remitidos por estudiantes de grado, posgrado o doctorado que, asesorados por un profesor-investigador, han desarrollado ejercicios de investigación de comprobado rigor. Siendo esta la primera revista de la UNAH que publica las investigaciones de estudiantes, ha tenido mayor impacto en el área de Ciencias Biológicas y de la Salud y en las carreras impartidas en UNAH en el Valle de Sula, en el norte del país. (Gráfico 2). Con el fin de fomentar la visibilidad de estos artículos científicos en el ámbito nacional e internacional y de facilitar su inclusión en diversos buscadores académicos, ambas revistas científicas se encuentran indexadas en Latindex y su contenido alojado en línea en Central American Journals Online (CAM$\mathrm{JOL})$.

Recientemente, se ha iniciado el proceso de indexación en DOAJ (Directory of Open Access Journals), ya que DOAJ aumenta la visibilidad, la accesibilidad, la reputación, el uso y el impacto de las revistas académicas de investigación de calidad, revisadas por pares y de acceso abierto a nivel mundial, independientemente de la disciplina, la geografía o el idioma. (DOAJ, 2003) De igual manera, se ha realizado un acercamiento con AmeliCA, para optar a indexación a través de ellos. AMELI, Conocimiento Abierto para América Latina y el Sur Global (AmeliCA) es una iniciativa puesta en marcha por la Organización de las Naciones Unidas para la Educación, la Ciencia y la Cultura (UNESCO), el Consejo Latinoamericano de Ciencias Sociales (CLACSO), la Red de Revistas Científicas de América Latina y el Caribe, España y Portugal (Redalyc), la Universidad Autónoma del Estado de México (UAEM), la Universidad de Antioquia (UdeA) y la Universidad Nacional de La Plata (UNLP). Se trata de una nueva configuración de estrategias, en respuesta al contexto internacional, regional, nacional e institucional, que busca una solución de Acceso Abierto colaborativa, sostenible, protegida y no comercial para América Latina y el Sur Global. (AmeliCA 2019) 
Gráfico 1. Participantes por facultad y centro regional en Revista Ciencia y Tecnología, UNAH 2007-2017

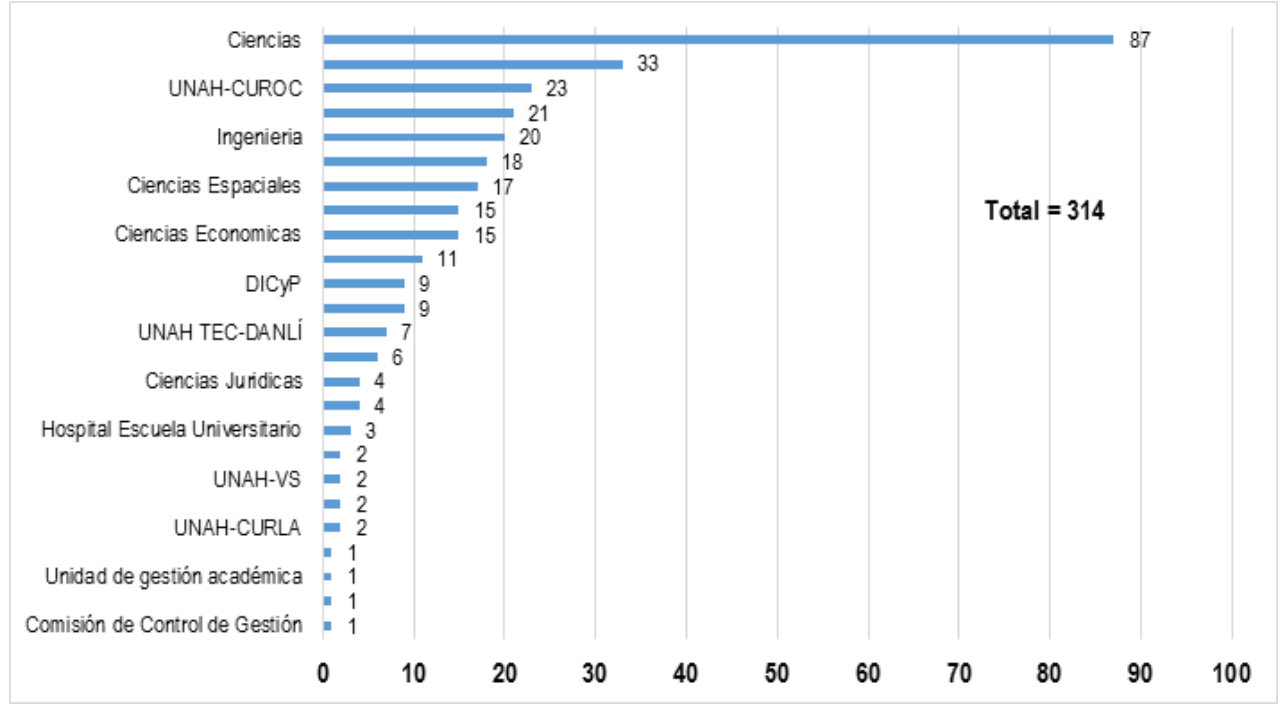

Fuente: Dirección de Investigación Científica, UNAH 2017

Gráfico 2. Participantes por facultad y centro regional en Revista Portal de la Ciencia, UNAH 2007-2017

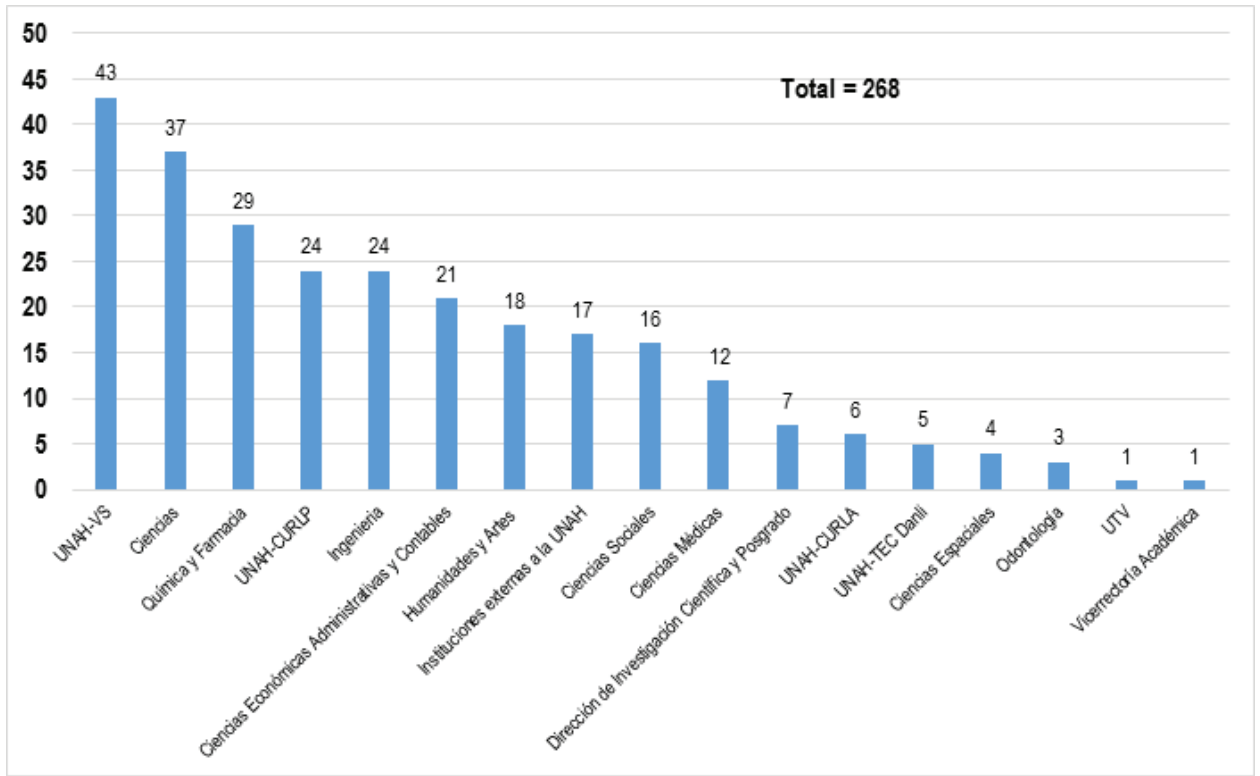

Fuente: Dirección de Investigación Científica, UNAH 2017 


\section{Publicación de artículos científicos generados por estudiantes de posgrado}

En conocimiento de la importancia de la publicación científica para el desarrollo científico y tecnológico, la UNAH utilizando como herramienta las nuevas Normas Académicas aprobadas en el año 2015, establece en el Artículo 25 del mismo, el requisito de producir al menos tres (3) artículos científicos desarrollados durante el proceso de elaboración de tesis como requisito para la obtención de un título de posgrado. En consecuencia, se perfila el desafío de proveer espacios institucionales para la publicación de artículos científicos que den cabida a las demandas de publicación de estudiantes de posgrado.

Actualmente existen 17 revistas científicas publicadas por unidades académicas de la UNAH, pero solamente 10 de ellas cuentan con ISSN y están indexadas en Latindex y tres de ellas están indexadas en LILACS. Se ha iniciado el capítulo Scielo-Honduras para albergar las revistas científicas que cumplen con la debida rigurosidad científica. La Red SciELO provee Acceso Abierto (AA) a los contenidos de revistas científicas. Las revistas se organizan en colecciones nacionales y temáticas. Cada colección es administrada por una organización científica reconocida a nivel nacional. La selección de revistas a ser indexadas o discontinuados en cada colección es de entera responsabilidad de la gestión de cada colección usualmente con el apoyo de un comité asesor científico. (SciELO,1997)

Las revistas disponibles para la publicación de artículos no son suficientes para albergar la producción esperada en los próximos años, por lo que se hace necesaria la creación de nuevas revistas científicas. En este sentido, las acciones de DICU se orientan hacia la puesta en marcha de iniciativas para el fortalecimiento de capacidades de redacción científica y evaluación de artículos científicos en el cuerpo de profesores universitarios.

La exigencia de tres artículos publicables por estudiantes de posgrado genera una oportunidad para elevar la producción científica de la UNAH, a través del fortalecimiento de la investigación en los posgrados siempre que se asegure que los profesores que imparten clases de posgrado sean investigadores. El profesor, que a su vez es investigador, velará por cuidar la calidad de los artículos científicos producidos desde su disciplina, ya que contará con las habilidades necesarias para asesorar metodológicamente al estudiante, evaluar la rigurosidad científica del manuscrito e incluso se asegurará de que el articulo publicable se inserte dentro de los 13 temas prioritarios de investigación de la UNAH y las líneas de investigación de su unidad académica. 
El profesor, además de dominar plenamente el contenido de su clase, debe permanecer en constante innovación, mejorando las prácticas pedagógicas y el desarrollo integral que el ejerce como docente. Para poder alcanzar su máximo potencial académico, el profesor debe de ser investigador para poder enfrentar y resolver problemas desde perspectiva de la ciencia, para poder motivar a sus alumnos a ser innovadores teniendo siempre una actitud positiva frente a la investigación como un medio para llegar al conocimiento y al aprendizaje significativo.

El profesor que realiza investigación, a su vez, contribuye a la formación de nuevos investigadores de forma integral, potenciando la capacidad de análisis y deducción, permitiéndoles desarrollar la observación y la reflexión. De igual forma, el profesor del posgrado contará con el apoyo de grupos de investigación para realizar las asesorías de tesis y evaluación de la calidad científica de los artículos que se producen.

Complementando lo anterior, la Dirección de Investigación Científica se encarga de dinamizar las iniciativas de creación de revistas en línea, gestionadas por grupos de programas de posgrado con cierta afinidad temática que, en conjunto, nombran a un equipo coordinador integrado por, al menos, un director, un comité editorial y un comité científico. Los profesores, garantizan el carácter científico de los artículos que se publicarán en dicha revista, al identificar las asignaturas en las que se desarrollarán los artículos científicos requeridos. La revista definirá sus objetivos generales, alcance y publico meta, todo en consonancia con las líneas de investigación definidas en la creación del posgrado, de acuerdo a su área temática.

\section{El catálogo de investigadores y sus publicaciones}

La Dirección de Investigación Científica ha identificado los investigadores que actualmente laboran en la UNAH, a través de su registro en línea en el Catálogo de Investigadores y sus publicaciones. Como parte del registro, los investigadores detallan las investigaciones realizadas en los últimos 3 años, definiéndolos de acuerdo a su tipo: artículos publicados en línea en revistas indexadas, artículos publicados en físico en revistas indexadas, publicaciones de capítulos de libros o libros completos, participaciones en congresos nacionales e internacionales de alto nivel científico y aquellos que tienen experiencia en asesoría de tesis, lo describen como tal. (Gráfico 3). 
Gráfico 3. Tipos de publicaciones por unidad académica según Catálogo de Investigadores UNAH 2015

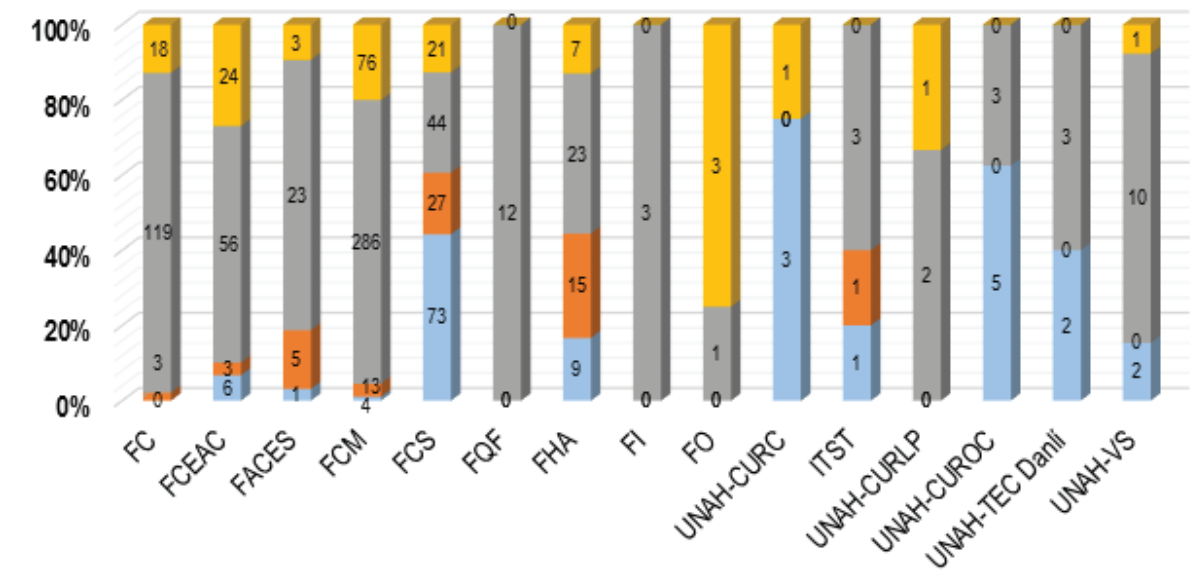

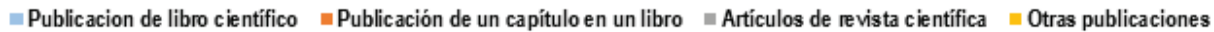

Fuente: Dirección de Investigación Científica, UNAH 2017

Este instrumento puede servir de base para poder identificar a los profesores de posgrado de acuerdo a su formación académica y formación en investigación, para que sean los mejor calificados los que emprendan la tarea de creación de revistas científicas y revisión de tesis (Gráfico 4.) Las instancias de publicación deben clasificarse de acuerdo a los niveles de investigadores que actualmente existen en la UNAH: para estudiantes de grado, estudiantes de posgrado y para profesores investigadores en formación, consolidación y de larga trayectoria. Esta clasificación fortalecerá la estructura interna de los posgrados, mejorando la calidad de la investigación que es realizada por los estudiantes. De esta manera, se estimula la producción de conocimiento científico desde el posgrado, se mejora la calidad de las revistas en donde ellos pueden publicar y se genera la creación de revistas que pueden pertenecer al primero, segundo, tercero o cuarto cuartil.

En materia de publicación científica se aumentará el número de revistas que pertenecen a la UNAH, ya que los posgrados tendrán mayor incidencia en crear sus propios espacios de publicación internos y al fortalecerse el apoyo para el fomento, la producción y la publicación del conocimiento científico, los profesores se estimularán más para la publicación de artículos científicos y divulgación de conocimiento a través de congresos científicos. 


\section{Gráfico 4. Total de investigadores y sus publicaciones por unidades académi- cas, según Catálogo de Investigadores, UNAH 2015}

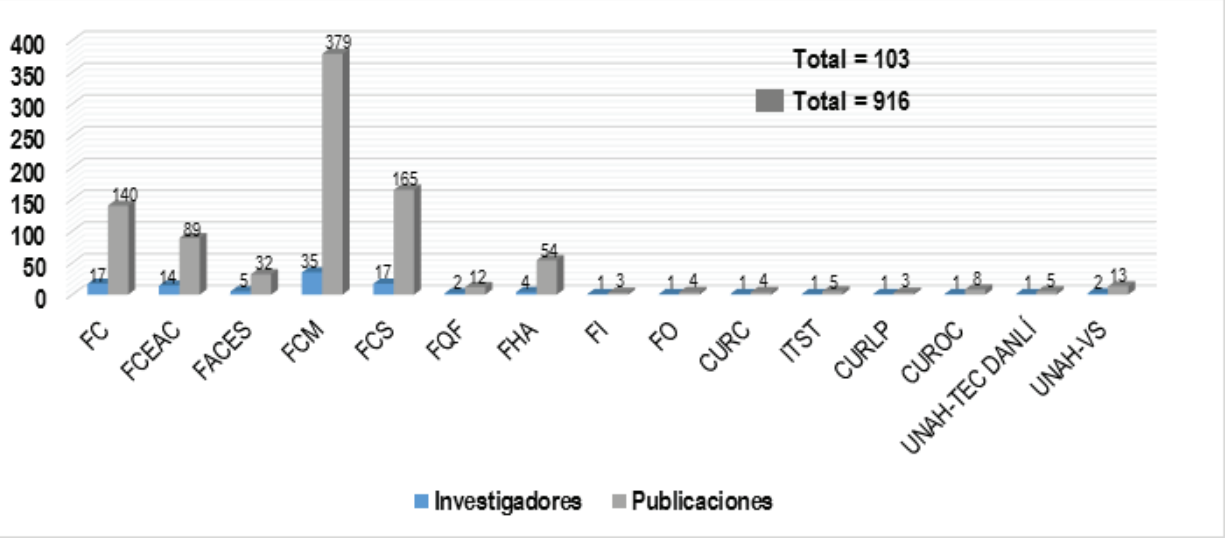

Fuente: Dirección de Investigación Científica, UNAH 2017

También se podrán potenciar las revistas que actualmente están en funcionamiento, pero que deben encaminarse a la publicación en línea principalmente; esta permite mayor visibilidad a nivel nacional, regional e internacional, provee alojamiento y respaldos seguros de los artículos, permite la indexación en Google Académico y amplía el desarrollo futuro de portales de revistas en línea por materia. Las revistas afines pueden desarrollar mecanismos colaborativos a nivel de la región centroamericana para la revisión de artículos por pares ciegos, ajenos a la entidad editora; igualmente, la revisión y publicación de artículos científicos de estudiantes de posgrado e investigadores hondureños en revistas de alto nivel y rigurosidad científica.

Curiosamente algunos investigadores con experiencia en publicación nacional, (Gráfico 5) ya no envían sus artículos a las revistas nacionales, solamente a revistas internacionales. Las revistas nacionales han tenido que desarrollar estrategias para resultar atractivas al investigador y captar originales de calidad a pesar de la migración de los autores hacia revistas extranjeras. Gráfico 6 . Es importante recordar que por encima de la obsesión por los factores de impacto, la revista más adecuada para publicar un documento dependerá de la audiencia a la que vaya dirigido. Publicar en las revistas con unos altos factores de impacto recogidos en las principales bases de datos y escritos en inglés, es decir, en revistas de alto prestigio internacional, puede llegar a ser en algunos casos contraproducente si dichas revistas no son las que leen los investigadores a los que van dirigidos los documentos. Como resultado de su escasa difusión entre los que serían sus máximos lectores y posteriores citantes, los 
documentos pueden recibir menos citas y producir, en definitiva, menos impacto del que hubieran causado de haber sido publicados en la lengua natal. (Bordons, 2004)

Grafico 5. Investigadores con más publicaciones nacionales, según unidad académica, UNAH 2015.

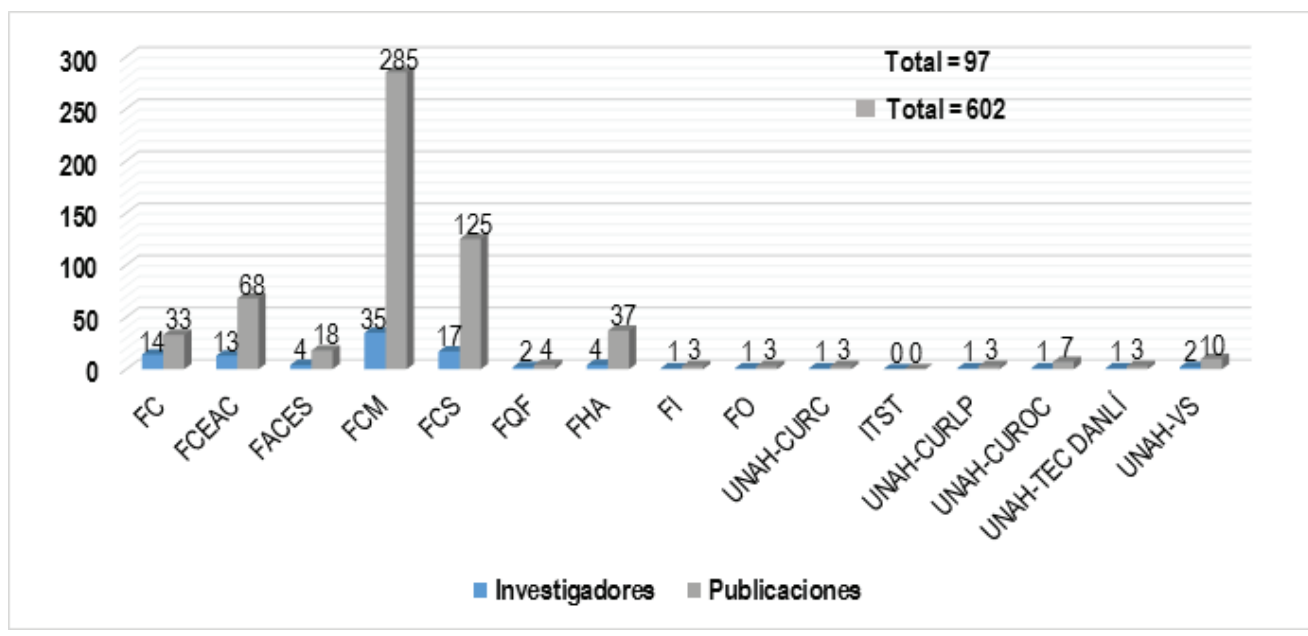

Fuente: Dirección de Investigación Científica, UNAH 2017

Gráfico 6. Investigadores y publicaciones internacionales, UNAH 2015.

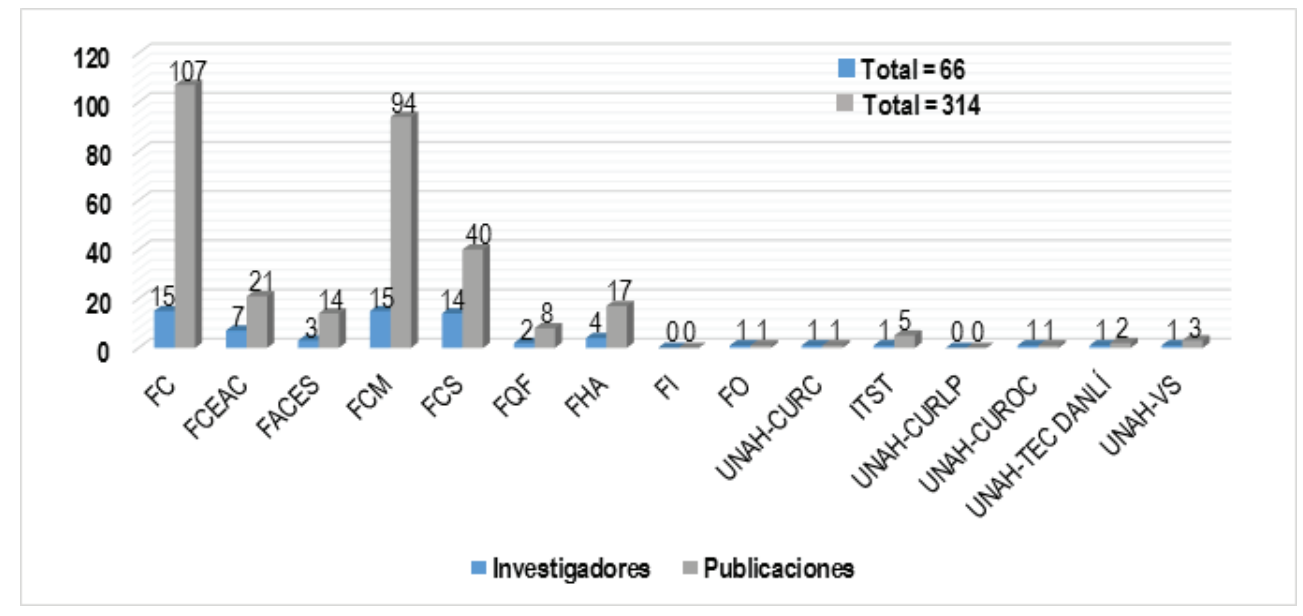

Fuente: Dirección de Investigación Científica, UNAH 2017 
Los posgrados en la UNAH han sido creados para resolver las necesidades del país de acuerdo a su oferta y demanda. Es por ello que se debe recordar el papel de la UNAH como universidad pública, que responde a la solución de los grandes problemas nacionales de acuerdo a la producción de conocimiento que genera el desarrollo de la ciencia y la tecnología. La publicación del conocimiento científico desde los posgrados responde a estas necesidades de solucionar los problemas básicos del país, por ello, debe fomentarse la producción y publicación de conocimientos científico a partir de la creación de revistas científicas en línea que cumplan con los estándares internacionales de rigurosidad científica establecidos por la comunidad científica a nivel nacional y regional.

\section{CONCLUSIONES}

a. Son los profesores universitarios los responsables de producir conocimiento científico y publicarlo en revistas de calidad, para dar respuesta a las prioridades de investigación institucional y nacional, y de esta manera contribuir a la resolución de problemas nacionales y regionales.

b. Para poder mejorar la publicación científica en la UNAH se debe trabajar arduamente en lo siguiente: creación de revistas en línea, que cumplan con los requisitos básicos de indexación internacional y fortalecer los lazos de comunicación con revistas regionales para mejorar el proceso de adquisición de artículos y revisión minuciosa de los mismos.

c. Identificar investigadores e instancias de publicación para los distintos niveles de investigadores: para estudiantes de grado, estudiantes de posgrado y para profesores investigadores en formación, consolidación y de larga trayectoria; así se podrán crear o fortalecer las instancias internas de la Universidad, instancias nacionales, regionales e internacionales y, dentro de estas, revistas de primero, segundo, tercero o cuarto cuartil.

\section{REFERENCIAS BIBLIOGRÁFICAS}

AmeliCA (2019) ¿Qué es AmeliCA? Recuperado de http://www.amelica.org/index.php/que-es-ameli/\#uno

Bordons, María . (2004) Hacia el reconocimiento internacional de las publicaciones científicas españolas. Revista Española de Cardiología.57(09). Centro de Información y Docu- 
mentación Científica (CINDOC). CSIC. Madrid. España.

Dirección de Investigación Científica y Posgrado, UNAH. (2015). Política de Investigación científica, desarrollo tecnológico e innovación UNAH 2015 - 2019. Recuperado de: <https://DICYP.unah.edu.hn/investigacion-cientifica/normativa/manuales/>.

Dirección de Investigación Científica y Posgrado, UNAH. (2015). Reglamento del Sistema de Investigación Científica y Tecnológica de la Universidad Nacional Autónoma de Honduras (SICYT). Recuperado de: https://DICYP.unah.edu.hn/investigacion- cientifica/normativa/manuales/>

Dirección de Investigación Científica Universitaria (2018). Todo en cifras 2007-2017. Recuperado de: https://dicu.unah.edu.hn/investigacion-cientifica/publicacion-comunicacion-ydifusion/todo-en-cifras/

DOAJ (2003). Misión de DOAJ. Recuperado de: https://doaj.org/about

SciELO. (1997) Declaración de Acceso Abierto. Recuperado de https://scielo.org/es/sobre-el-scielo/declaracion-de-accesso-abierto/ 